\title{
Prenatal choline supplementation alters the timing, emotion, and memory performance (TEMP) of adult male and female rats as indexed by differential reinforcement of low-rate schedule behavior
}

\author{
Ruey-Kuang Cheng, Christopher J. MacDonald, Christina L. Williams, \\ and Warren H. Meck ${ }^{1}$ \\ Department of Psychology and Neuroscience, Duke University, Genome Sciences Research Building II, \\ Durham, North Carolina 27708, USA
}

\begin{abstract}
Choline availability in the maternal diet has a lasting effect on brain and behavior of the offspring. To further delineate the impact of early nutritional status, we examined effects of prenatal-choline supplementation on timing, emotion, and memory performance of adult male and female rats. Rats that were given sufficient choline (CON: 1.1 $\mathrm{g} / \mathrm{kg}$ ) or supplemental choline (SUP: $5.0 \mathrm{~g} / \mathrm{kg}$ ) during embryonic days (ED) 12-17 were trained with a differential reinforcement of low-rate (DRL) schedule that was gradually transitioned through 5-, 10-, 18-, 36-, and 72-sec criterion times. We observed that SUP-females emitted more reinforced responses than CON-females, which were more efficient than both groups of males. In addition, SUP-males and SUP-females exhibited a reduction in burst responding (response latencies $<2 \mathrm{sec}$ ) compared with both groups of CON rats. Furthermore, despite a reduced level of burst responding, the SUP-males made more nonreinforced responses prior to the DRL criterion as a result of maintaining the previous DRL criterion following transition to a new criterion. In summary, long-lasting effects of prenatal-choline supplementation were exhibited by reduced frustrative DRL responding in conjunction with the persistence of temporal memory in SUP-males and enhanced temporal exploration and response efficiency in SUP-females.
\end{abstract}

Choline is an essential nutrient for the regulation of cell signaling, methyl metabolism, and acetylcholine synthesis in both the infant and adult (Zeisel and Niculescu 2006). It has been found that when choline is administered at levels higher than normal to pregnant rats during embryonic days (ED) 12-17, the adult offspring showed enhanced spatial and temporal memory (Meck and Williams 2003). Specifically, rats given this prenatal-choline supplementation made fewer errors when tested on a radial-arm maze (Meck et al. 1988, 1989; Meck and Williams 1999) and are able to chunk more information in spatial memory that helps them identify multiple-reward baiting patterns (Dallal and Meck 1990; Meck and Williams 1997b). Similarly, when challenged with tasks that require the ability to remember durations in the seconds-to-minutes range, prenatal choline-supplemented rats produced more precise timing functions and are better able to time multiple durations simultaneously compared with control rats (Meck and Williams 1997a,c; Cheng et al. 2006b). The prenatal availability of choline also modifies the development of the cholinergic system (e.g., Meck et al. 1989; Williams et al. 1998; Cermak et al. 1999; Montoya et al. 2000; Mellott et al. 2004) and alters indices of hippocampal plasticity (e.g., Pyapali et al. 1998; Glenn et al. 2007). Together, these findings indicate that prenatal-choline supplementation can induce profound changes in brain function that lead to increased memory capacity and precision in adult offspring. In the present study, the potential benefits of prenatal-choline supplementation were further investigated by using differential reinforcement of low-rate (DRL) schedules to study the timing, emotion, and memory perfor-

\footnotetext{
1 Correspondence author.
}

E-mail meck@psych.duke.edu; fax (919) 660-5798.

Article is online at http://www.learnmem.org/cgi/doi/10.1101/lm.729408. mance (TEMP) of adult male and female rats (Matell and Meck 2000, 2004; MacDonald and Meck 2004; Buhusi and Meck 2005; Droit-Volet and Meck 2007; Meck and MacDonald 2007).

During the typical DRL schedule, a rat is trained to withhold lever pressing for a minimum amount of time following the previous lever press, in order to earn reinforcement. Responses emitted before the criterion time are not reinforced, but instead reset the experimental clock, forcing the subject to withhold responding again until the criterion time has passed. Hence, this schedule can access both the self-paced timing ability of subjects, as well as their capacity to inhibit impulsive responding following errors (e.g., Richards et al. 1993; Evenden 1999; Ripley et al. 2001). We utilized a series of DRL schedule values in order to study emotional behavior as indexed by premature burst responding, as well as memory endurance indexed by the resistance to change during the transition from one criterion time to another. This was accomplished by first training rats to learn short DRL criteria (e.g., 5 and $10 \mathrm{sec}$ ) and then transitioning them to progressively longer criteria up to a maximum of $72 \mathrm{sec}$. This shifting of DRL schedule values is particularly useful for examining memory endurance and/or resistance to change, primarily because of the reset of the experimental clock following premature responses. For example, if a rat remembered the previous DRL criterion (e.g., $18 \mathrm{sec}$ ) so strongly that it keeps pressing the lever around that time even after the transition to a longer criterion (e.g., $36 \mathrm{sec}$ ), the clock-reset design inherent in the DRL schedule will impede it from coming into contact with the reinforcement contingency of the new criterion.

In order to characterize the effect of memory endurance in DRL schedules, we monitored changes in the modified response efficiency as a function of training sessions during DRL training at each temporal criterion. Modified response efficiency is de- 
fined as the ratio between reinforcement rate and overall response rate after the subtraction of burst responses-defined by lever presses with an inter-response time (IRT) $<2$ sec. Burst responding was excluded in determining the modified response efficiency, because previous studies have suggested that these rapid responses are more related to behavioral inhibition and emotional reactivity (e.g., frustration, impulsivity, and the failure of self-control) than to timing or temporal memory per se (e.g., Wiley et al. 2000; Cheng et al. 2006a). Therefore, it seems reasonable to dissociate burst responding from response efficiency and to treat both measures independently when evaluating performance on DRL schedules. Finally, in order to investigate the acquisition of each DRL criterion, performance during the initial, intermediate, and final phases of training were treated as main factors (i.e., training phase-three levels) when analyzing the behavioral data for longer DRL criteria (i.e., 18, 36, and $72 \mathrm{sec}$ ).

In summary, adult male and female rats that were treated with either prenatal-choline supplementation (SUP) or a control $(\mathrm{CON})$ level of choline, were trained in a series of DRL schedules with transitions from 5 to $72 \mathrm{sec}$. Our hypothesis was that prenatal-choline supplementation would enhance the cognitive processes that distinguish males and females and thereby accentuate the normal sex differences observed in exploratory behavior (Tropp and Markus 2001). Consequently, it was our expectation that SUP-males would be less exploratory and more resistant to changes in the temporal criteria and maintain the previous DRL criterion. In contrast, the SUP-females would demonstrate increased "temporal exploration", reflected by the expansion of their IRT distributions in response to changes in the interfood intervals and therefore acquire a new criterion more rapidly (see Church et al. 1998).

\section{Results}

\section{DRL 5-sec schedule training}

During the last session of DRL 5-sec acquisition, the two-way ANOVAs indicated a significant main effect of sex on modified response efficiency $\left(F_{(1,2)}=5.18 ; P<0.05\right.$; see Fig. $\left.1 \mathrm{~A}\right)$ and on burst responses $\left(F_{(1,28)}=4.85 ; P<0.05\right.$; see Fig. $\left.1 \mathrm{~B}\right)$. The post-hoc tests showed that female rats displayed a higher modified response efficiency $(P<0.05)$ and a lower level of burst responding $(P<0.05)$ than did male rats. No significant effects of choline treatment were observed (for $P^{\prime} \mathrm{s}>0.05, \mathrm{MSE}=0.007$ and 634.8 for response efficiency and burst responding, respectively).

\section{DRL 10-sec schedule training}

During the last session of DRL 10-sec acquisition, the two-way ANOVAs indicated significant main effects of both sex $\left(F_{(1,28)}=5.11 ; P<0.05\right)$ and choline treatment $\left(F_{(1,28)}=4.23\right.$; $P<0.05$ ) on modified response efficiency (see Fig. $1 C$ ). There was also a significant main effect of sex on burst responding $\left(F_{(1,28)}=6.14 ; P<0.05\right.$; see Fig. 1D). The post-hoc tests showed that female rats had a higher modified response efficiency than male rats $(P<0.05)$, while the prenatally choline supplemented rats showed a lower modified response efficiency compared with control rats $(P<0.05)$. In terms of burst responding, female rats again showed lower levels of burst responding than male rats $(P<0.05)$.

\section{DRL 18-sec schedule training}

Three-way ANOVAs were conducted on the behavioral data obtained from DRL 18-sec to DRL 72-sec training by using sex, choline treatment, and training phase as three different factors. During DRL 18-sec training (see Figs. 2A, 3A), the ANOVA indicated significant main effects of sex $\left(F_{(1,28)}=21.89 ; P<0.001\right)$ and training phase $\left(F_{(2,56)}=105.63 ; P<0.001\right)$ on modified response efficiency. The post-hoc test revealed that female rats showed higher levels of modified response efficiency than male rats $(P<0.001)$. In terms of training, post-hoc comparisons indicated that the modified response efficiency during intermediate and late training phases were both significantly higher than during the early phase $(P<0.001)$ and that response efficiency during the intermediate and late phases did not differ (see Fig. 4A).

With regard to burst responding, the ANOVA indicated significant main effects of $\operatorname{sex}\left(F_{(1,28)}=10.16 ; P<0.01\right)$ and training phase $\left(F_{(2,56)}=13.68 ; P<0.001\right)$. In this case, there was no main effect of choline treatment on burst responding $\left(F_{(1,28)}=3.19\right.$; $P<0.08)$. However, a significant sex $\times$ training interaction was
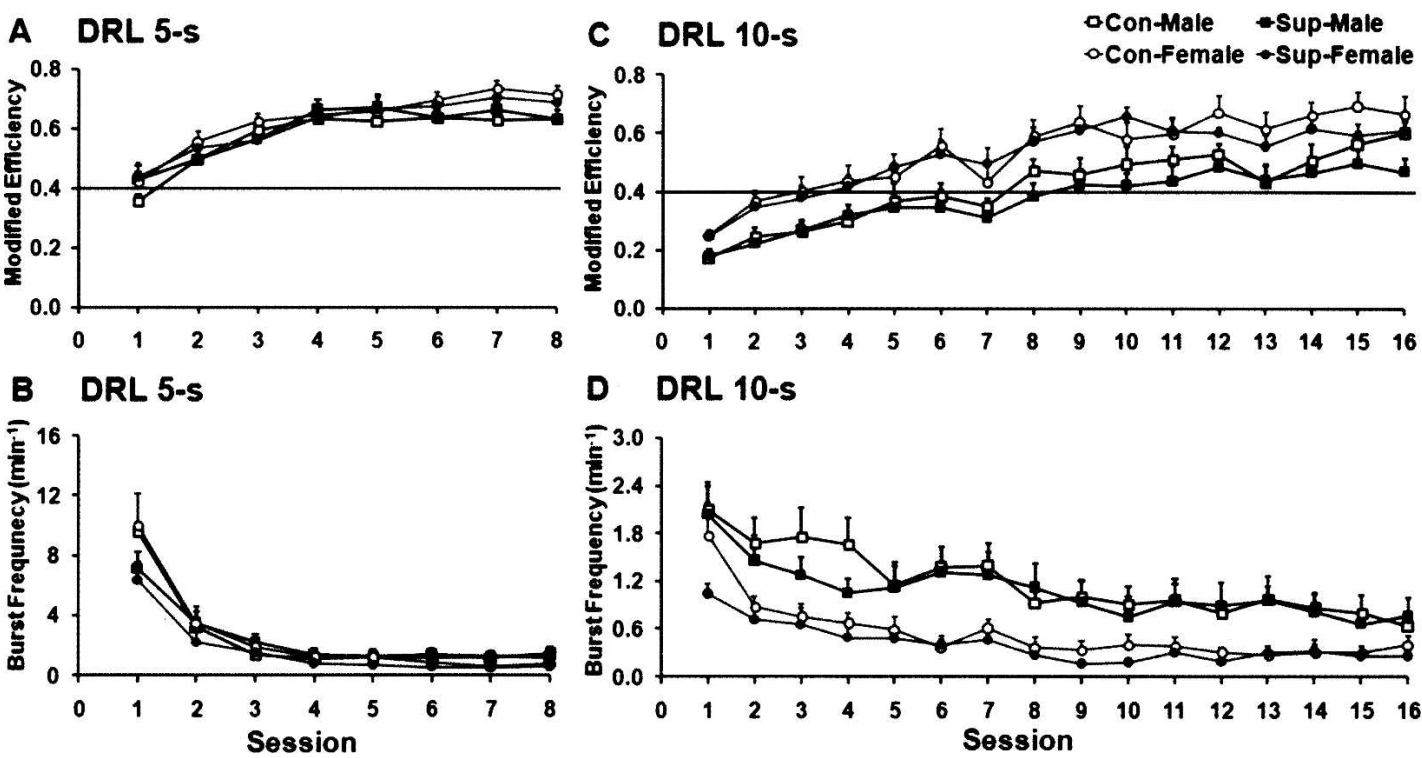

Figure 1. Mean (+SEM) modified response efficiency $(A, C)$ and burst-responding rate $(B, D)$ plotted as a function of sessions during the DRL 5 -sec and DRL 10-sec schedule training stages. Modified response efficiency is determined by a ratio between reinforcement rate and overall responding rate (after subtraction of burst responding rate). Burst-responding rate is determined by the average occurrence of burst responding per minute in a session. 


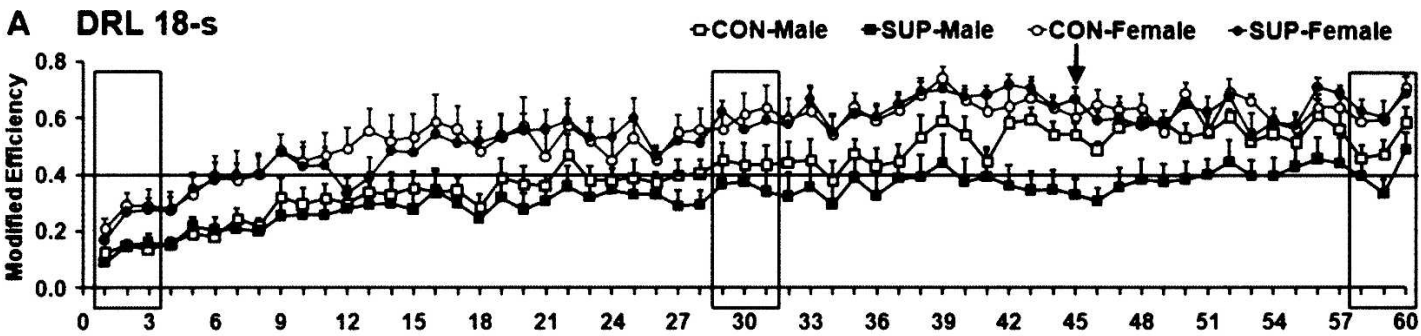

B DRL 36-s

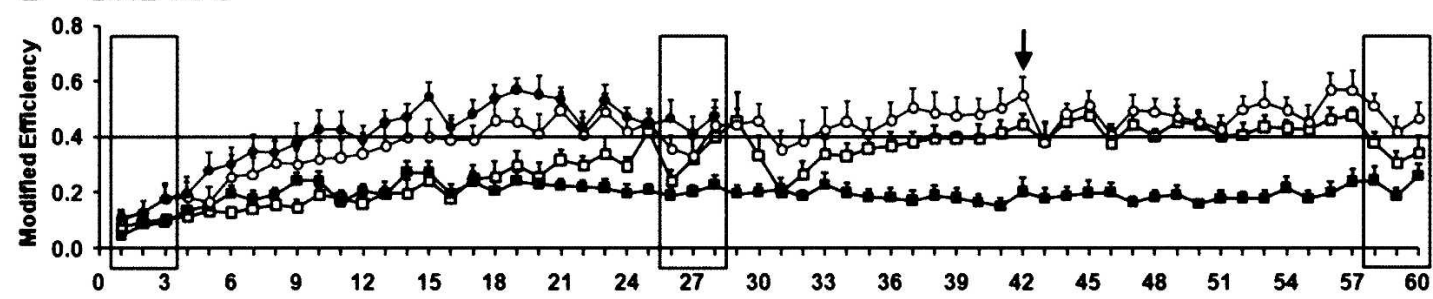

C DRL 72-s

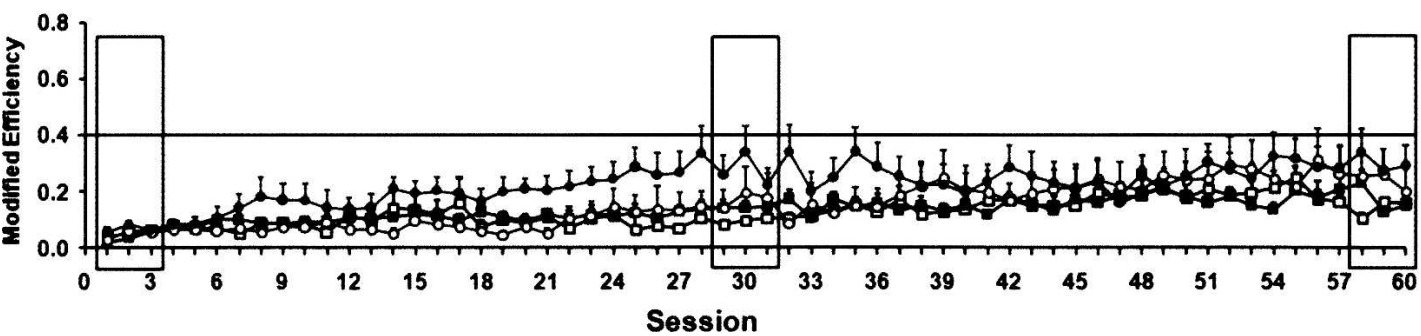

Figure 2. Mean (+SEM) modified response efficiency plotted as a function of sessions during the DRL 18-sec (A), DRL 36-sec (B), and DRL 72-sec (C) training stages. The individual rectangles in each panel indicate the three sessions that are averaged to represent the three training phases (i.e., early, intermediate, and late phases). The means taken from the three training phases are used for statistical analysis depicted in Figure 4, left. Arrows in $A$ and $B$ indicate the sampled sessions that are further examined with the inter-response times (IRT)-distribution and peak time analyses presented in Figures 5 and 6 , respectively.

observed $\left(F_{(2,56)}=3.69 ; P<0.05\right)$. Post-hoc tests showed that female rats had lower levels of burst responding than male rats $(P<0.01)$, while the level of burst responding during the early phase of DRL training was higher $(P<0.001)$ than the level during the intermediate and late phases, which did not differ from each other (see Fig. 4B). The interaction between sex and training can best be explained by the results of the post-hoc comparisons, indicating that male rats showed a decrease in burst responding during the intermediate and late phases of training compared with the early phase (both $P^{\prime} s<0.001$ ), while the level of burst responding did not differ between these two phases. This type of interaction was not observed in female rats (see Fig. 4B).

\section{DRL 36-sec schedule training}

During DRL 36-sec training (see Figs. 2B, 3B), one exception to our training procedures was that the SUP-female rats were shifted to DRL 72-sec training after 28 sessions of DRL 36-sec training, because all of the subjects achieved a high level of modified response efficiency $(>0.40)$ by that time. Hence, we pooled the behavioral data collected during sessions $26-28$ in order to represent performance during the intermediate training phase for all four treatment groups on the DRL 36-sec schedule. Given that the SUP-female rats already performed at a high level of efficiency after 28 sessions of DRL 36-sec training, the performance of SUP-female rats during the intermediate phase was taken as representative of their performance during the late phase. This was done in order to preserve three levels of training phase across all four treatment groups for conducting subsequent ANOVAs.
The ANOVA indicated significant main effects of sex $\left(F_{(1,28)}=13.08 ; P<0.01\right)$ and training phase $\left(F_{(2,56)}=83.58\right.$; $P<0.001)$, accompanied by a significant sex $\times$ training interaction $\left(F_{(2,56)}=3.83 ; P<0.03\right)$ for the modified response efficiency. Post-hoc tests revealed that female rats had a higher modified response efficiency than male rats $(P<0.01)$ and that the modified response efficiency during the early phase of training was significantly lower than during the intermediate and late phases $(P<0.001)$, which did not differ from each other $(P<0.05)$. A post-hoc comparison of the sex $\times$ training interaction revealed that the modified response efficiency of female rats increased more than male rats during both the intermediate and late phases of training; $P<0.05$ and $P<0.01$, respectively (see Fig. 4C).

With regard to burst responding, the ANOVA indicated significant main effects of both choline treatment $\left(F_{(1,28)}=8.17\right.$; $P<0.01)$ and training phase $\left(F_{(2,56)}=17.21 ; P<0.001\right)$. The posthoc comparison revealed that rats that received prenatal-choline supplementation displayed lower levels of burst responding than control rats $(P<0.01)$. In terms of training phase, a similar pattern was observed in that burst responding during the early phase were significantly higher than during the intermediate and late phases $(P<0.001)$, while these two phases did not differ from each other, $P<0.05$ (see Fig. 4D).

\section{DRL 72-sec schedule training}

During DRL 72-sec training (see Figs. 2C, 3C), one SUP-male rat and one $\mathrm{CON}$-male rat were excluded from the analysis because of health problems. The ANOVAs indicated a significant main 

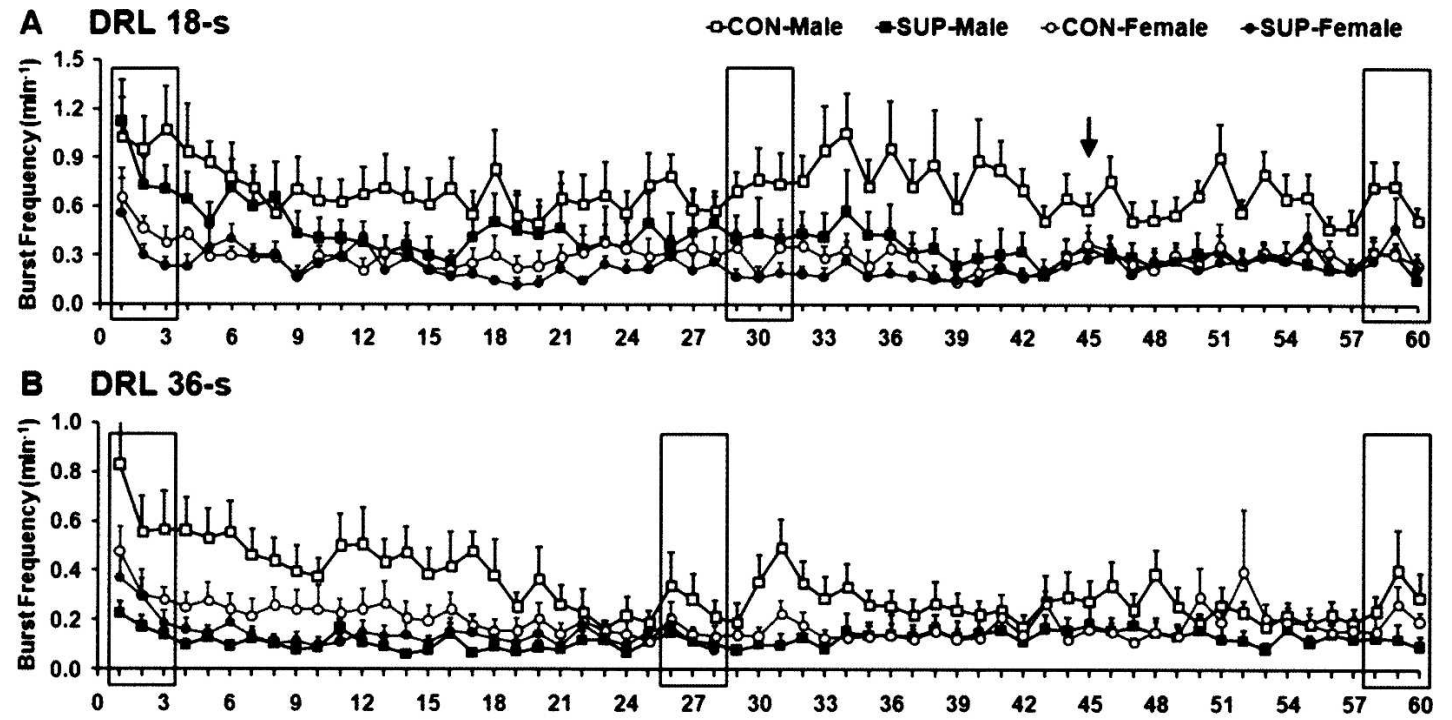

C DRL 72-s

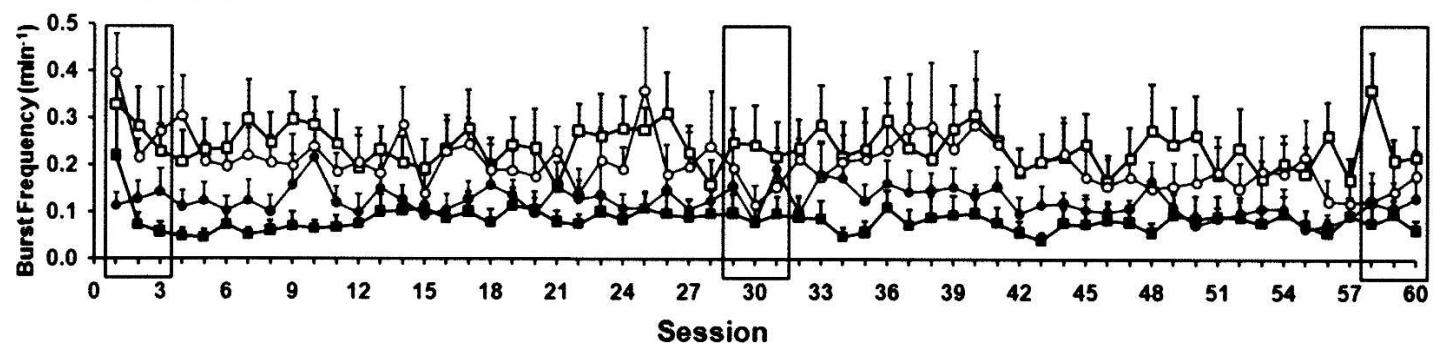

Figure 3. Mean (+SEM) burst-responding rate plotted as a function of sessions for DRL 18-sec (A), DRL 36-sec (B), and DRL 72-sec ( $C$ ) schedule training stages. The means from the three rectangles in each panel are used for statistical analysis depicted in Figure 4, right. The arrow in $A$ indicates the session selected for the burst-ratio analysis presented in Figure 7.

effect of training phase $\left(F_{(2,52)}=21.45 ; P<0.001\right)$ on the modified response efficiency, while the effect of sex was nonsignificant $\left(F_{(1,26)}=3.52 ; P=0.07\right)$. Post-hoc tests showed that during the early phase of training the modified response efficiency was significantly lower than during intermediate and late phases of training $(P<0.001)$, which did not differ from each other, $P<0.05$ (see Fig. 4E). In terms of burst responding, the ANOVA indicated significant main effects of choline $\left(F_{(1,26)}=6.83\right.$; $P<0.05)$ and training phase $\left(F_{(2,52)}=3.97 ; P<0.05\right)$. The posthoc comparison revealed that rats that received prenatal-choline supplementation displayed a lower level of burst responding than control rats $(P<0.05)$. Burst responding during the early phase of training were significantly higher in number than during the intermediate and late phases of training, which did not differ from each other, $P<0.05$ (see Fig. $4 \mathrm{~F}$ ).

Peak time analysis of DRL 18-sec and DRL 36-sec schedule performance

Atypical inter-response time (IRT) patterns were observed in association with the low levels of modified response efficiency reported for the SUP-male rats-especially during the intermediate phase of DRL 18-sec and DRL 36-sec schedule training (see Fig. $2 \mathrm{~A}, \mathrm{~B})$. Because the results of the ANOVAs conducted on the modified response efficiency measures indicated that SUP-male rats did not show significant improvement after an intermediate amount of training, the performance between the intermediate and the late phases can actually be considered steady-state performance. Therefore, we sampled one session between the intermediate and late phases during DRL 18-sec and during DRL 36-sec training. Further analysis of these two sessions (as indicated by the arrows in Fig. 2A,B) were used to assist the analysis of this unusual timing performance of the SUP-male rats. IRT frequency distributions that characterize lever presses with an IRT $>2 \mathrm{sec}$, but less than two times the criterion duration (i.e., 36 or $72 \mathrm{sec}$ ) as well as the mean peak times for each treatment group determined by a sliding-window method (see statistical analyses section), are plotted in Figure 5 for DRL 18-sec and in Figure 6 for DRL 36-sec schedule performance, respectively.

For peak times obtained from the sample DRL 18-sec session, a two-way ANOVA indicated significant main effects of sex $\left(F_{(1,28)}=12.99 ; P<0.01\right)$ and a significant sex $\times$ choline treatment interaction $\left(F_{(1,28)}=7.10 ; P<0.05\right)$. Post-hoc comparisons revealed that the peak times of female rats were significantly longer than male rats $(P<0.01)$, while the peak time of the SUPmale rats was significantly shorter than for all other treatment groups $(P$ 's $<0.05)$. For peak times obtained from the sample DRL 36-sec session, a one-way ANOVA using the three groups of rats that were actually trained in that session (i.e., CON-male, SUPmale, and CON-female groups) indicated a significant main effect of treatment group $\left(F_{(2,21)}=4.56 ; P<0.05\right)$. Post-hoc comparisons showed that the peak times of the SUP-male rats were significantly shorter compared with the other two groups of rats $\left(P^{\prime} \mathrm{s}<0.05\right)$.

\section{Analysis of burst ratio in DRL 18-sec schedule performance}

In order to characterize the burst reduction effect of prenatalcholine supplementation in SUP-male rats, two IRT windows 

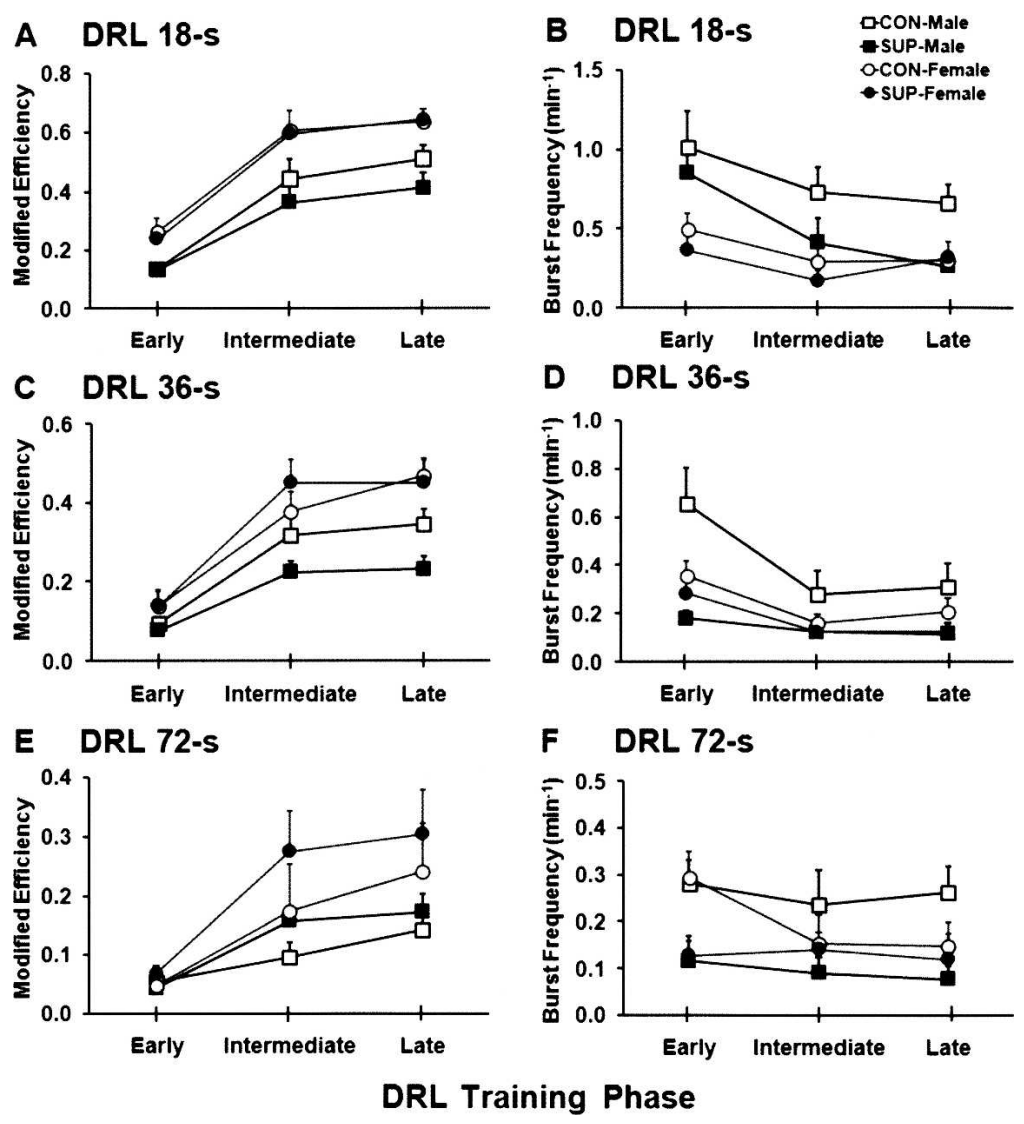

Figure 4. Mean (+SEM) modified response efficiency $(A, C, E)$ and burst-responding rate $(B, D, F)$ plotted as a function of training phases for DRL 18-sec, DRL 36-sec, and DRL 72-sec schedules. The data points for the DRL 36-sec schedule from the SUP-female rats in the late training phase are duplicated from their data points in the intermediate training phase. This is because the SUP-female group was the only treatment group in which all rats achieved threshold-level performance (modified response efficiency $>0.4$ ) and they were shifted to the DRL 72-sec schedule after an intermediate amount of training with the DRL 36 -sec schedule.

(8-10-sec bins and 14-18-sec bins) from the mean frequency IRT distribution (see Fig. 7A) were selected for burst ratio analysis as shown in Figure 7. In these two time windows, the total number of lever presses and the subsequent burst responding (IRT $<2 \mathrm{sec}$ ) were both recorded and calculated as a ratio in order to reflect the probability of a rat emitting a burst response when it pressed the lever during a specific IRT window. A two-way ANOVA indicated significant main effects of choline treatment $\left(F_{(1,14)}=9.66\right.$; $P<0.01)$ and each of the two time windows $\left(F_{(1,14)}=9.84\right.$; $P<0.01)$, whereas the choline $\times$ time window interaction was nonsignificant $\left(F_{(1,14)}=3.70 ; P=0.07\right)$. Post-hoc comparisons indicated that the SUP-male rats demonstrated lower burst ratio for the two IRT time windows compared with CON-male rats $(P<0.01)$. The burst ratios observed for these two time windows were also significantly different from each other $(P<0.01)$, indicating that both treatment groups showed an increase in the burst ratio from the first to the second time window, which was placed just before the DRL criterion time. In particular, the CONmale rats showed the largest increase in their burst ratios during the second time window compared with SUP-male rats $(P<0.01)$.

\section{Discussion}

Three major findings were observed in the present study. First, during each of the DRL training criteria, female rats exhibited a higher modified response efficiency than male rats. In addition, antidepressant drugs (Cohen et al. 1997; Louis et al. 2006). Consequently, one can consider burst responding as an expression of frustration to the "penalty" of reinforcement omission and clock reset. In contrast, subjects that emit fewer burst responses have the potential to be more efficient because they have additional opportunities to earn reinforcement. The reduction in burst responding observed for SUP-male rats in the present study is the first evidence showing that prenatal choline supplementation is beneficial to adult rats under situations in which the expression of emotional behavior is counterproductive to efficient performance. Hence, in addition to the enhancement of timing and temporal memory (Meck and Williams 1997a), prenatal-choline supplementation assists in the regulation of frustration and impulsivity as evidenced by reduced burst responding in DRL schedules.

In terms of response efficiency, an interaction between sex and prenatal-choline supplementation was observed. The general finding is that SUP-male rats were less efficient than CON-male rats, while, in contrast, SUP-female rats were more efficient than CON-female rats (Fig. 2). This sex difference is consistent with previous studies reporting that female rats are more efficient than male rats in DRL schedules of reinforcement (e.g., Beatty 1973; van Hest et al. 1987). This effect can be abolished by removal of circulating ovarian steroids, such that ovariectomized females perform equally to intact and castrated males, which did not differ from each other (Beatty 1973). Pregnancy and replacement 

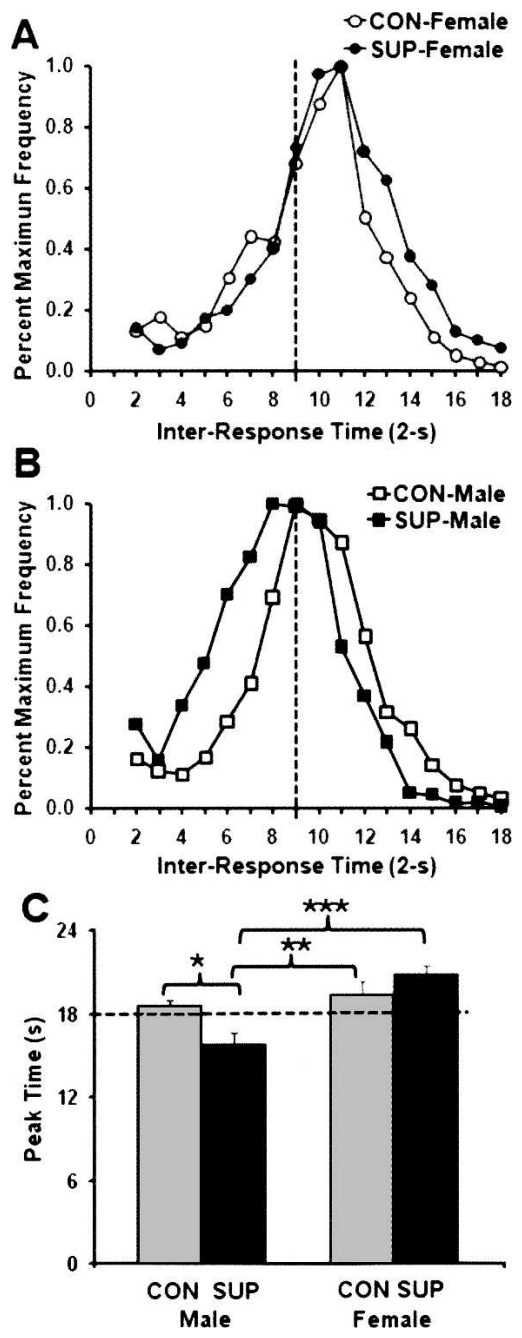

Figure 5. Mean percent maximum frequency of responding as a function of inter-response times (IRTs) plotted in 2-sec time bins during session-45 of the DRL 18-sec schedule for female rats $(A)$ and male rats $(B)$. Mean (+SEM) peak time (sec) for male and female rats as a function of prenatal choline treatments are plotted in C. Significance levels in comparison with SUP-male rats are indicated: ${ }^{*} P<0.05 ;{ }^{*} P<0.01$; ${ }_{* * *} P<0.001$.

of progesterone to ovariectomized females rats improves their DRL efficiency, suggesting that the efficient DRL-schedule performance of female rats can be attributed, in part, to circulating progesterone (e.g., Molina-Hernandez et al. 2000). The current finding that the SUP-female rats were even more efficient when acquiring longer DRL criterion times (e.g., 36 and $72 \mathrm{sec}$ ) implies that there is an interaction between prenatal-choline supplementation and gonadal hormones, possibly estrogen or progesterone, in female rats (Ross and Santi 2000).

An especially intriguing finding in the current study was the observation that SUP-male rats displayed sustained low-level response efficiency (Fig. 2) and yet had fewer burst responses compared with CON-male rats (Fig. 3). This is unusual because previous studies have shown that a reduction in DRL efficiency is usually accompanied by an increase in burst responding, a pattern that can be induced by administration of psychostimulants (e.g., Cheng et al. 2006a). The prevailing explanations for this pattern of responding are either that these drugs accelerated the speed of an internal clock and/or increased impulsivity, which in turn would decrease response efficiency (e.g., Meck 1996; Wiley et al. 2000; McAuley et al. 2006; Matell and Portugal 2007). Both of these explanations are likely to be inappropriate in the present situation because of the observation that SUP-male rats display the same temporal accuracy with increased precision compared with CON-male rats when assessed in other reproduction tasks such as the peak-interval procedure (e.g., Meck and Williams 1997a; Cheng et al. 2006b; Cheng and Meck 2007). In addition, the observation that SUP-male rats made fewer burst responses following premature responses that were close to the criterion time refutes the idea of increased impulsivity. Together with the finding that the SUP-male rats maintained low levels of response efficiency for $\sim 30$ consecutive sessions regardless of the training experience, we propose that this response pattern should be attributed to effects on temporal memory and response persistence instead of arousal effects on clock speed (see Cheng et al. 2007a,b; Meck 1983, 1988, 2002; Meck et al. 1986, 1987). Because circulating testosterone is known to increase persistence (Archer 1977), perhaps prenatal-choline supplementation enhances this hormonal effect.

Another possible explanation for the unusual behavior of

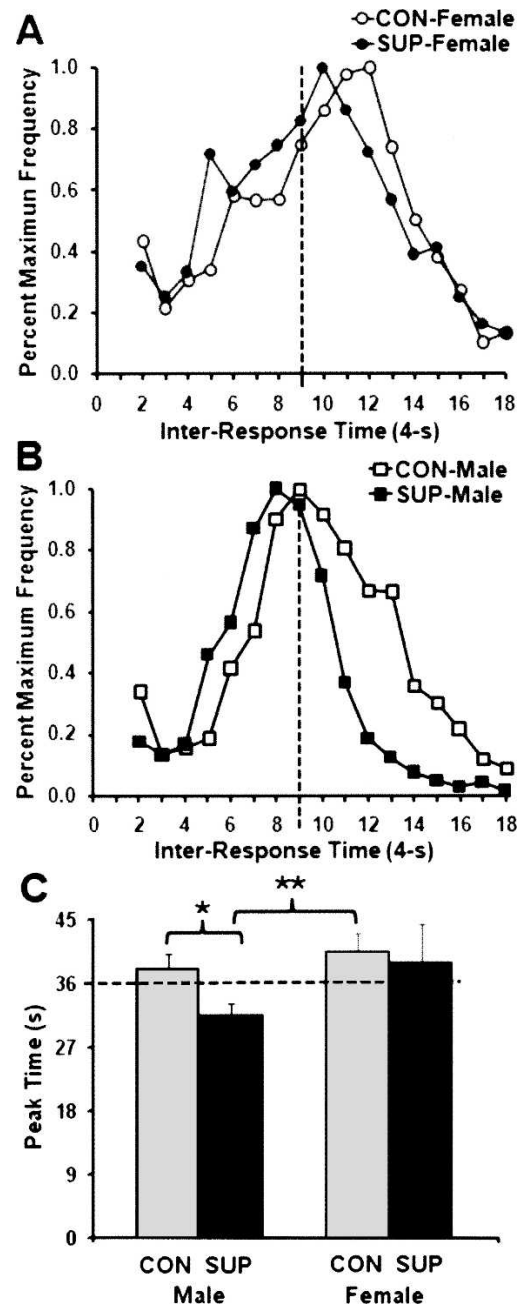

Figure 6. Mean percent maximum frequency of responding as a function of inter-response times (IRTs) in 4-sec time bins during session-42 of the DRL 36-sec schedule for female rats $(A)$ and male rats $(B)$ as a function of prenatal choline availability. Mean (+ SEM) peak times (sec) for male and female rats as a function of prenatal choline treatments are plotted in C. Significance levels in comparison with SUP-male rats are indicated: ${ }^{\star} P<0.05 ;{ }^{* \star} P<0.01$. 

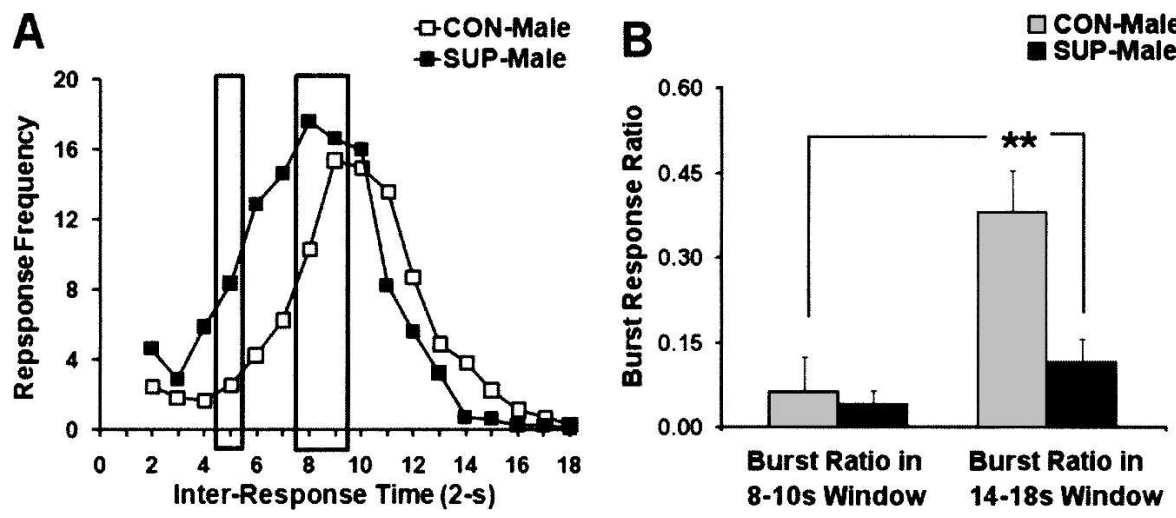

Figure 7. Mean frequency of responding as a function of inter-response times (IRTs) plotted in 2-sec time bins during session-45 of the DRL 18-sec schedule for male rats $(A)$. The two rectangles represent the sample windows (8-10 sec bins and 14-18 sec bins) for burst-ratio analysis. Mean (+SEM) burstresponding ratio for male rats plotted as a function of the two sampled time windows $(B)$. The significance level for the comparison between CON- and SUP-male rats is indicated: ${ }^{* *} P<0.01$.

SUP-male rats is that the general level of activity between IRTs can determine the overall DRL efficiency. In a study by van Hest et al. (1987), male rats that showed lower activity levels in an open-field test were also less efficient in DRL schedules compared with female rats. The authors inferred that male rats are generally less active than female rats, so that they have lower levels of "interim behaviors" during the waiting period, which in turn led to poor DRL efficiency. This account does not fit the present situation, because highly active male rats are no more efficient (and sometimes even less) in DRL schedules of reinforcement (e.g., Bardo et al. 2006; van den Bergh et al. 2006). Furthermore, recent studies have demonstrated that prenatal-choline supplementation facilitates open-field exploration as well as object exploration in both male and female rats, while producing no differences in spontaneous activity (Meck and Williams 2008). Hence, there is no indication that SUP-male rats are less active than CON-male rats, as measured by a variety of activity measures.

We hypothesize that the sustained low-level response efficiency observed in SUP-male rats can be explained by proposing that prenatal-choline supplementation leads to the development of a memory system that enables rats to form more enduring memories. This effect of memory endurance can be more readily evaluated by the use of DRL schedules (compared with other duration reproduction procedures; see Paule et al. 1999) because of the reinforcement contingency that requires responses made before the criterion time to reset the experimental clock. Because of this interval-reset property, rats that form a more enduring memory for shorter criterion durations (e.g., $18 \mathrm{sec}$ ) will continue to press the lever around that original criterion duration and be less efficient in acquiring longer criterion durations (e.g., $36 \mathrm{sec}$ ) in DRL schedules. This idea is similar to the "resistance to extinction" hypothesis proposed to account for variations in the strength of responding engendered by different schedules of reinforcement (e.g., Nevin et al. 2001). Compared with the memory endurance and temporal exploration for sequentially trained criterion times, parallel observations have been made using the Morris water maze, in which rats are inferred to have better memory for the spatial location of a hidden platform if they spend more time during extinction/probe trials exploring the location that once contained the platform (e.g., Schulz et al. 2004). In fact, there is a variety of neurobiological evidence supporting the view that rats given prenatal-choline supplementation are capable of forming more enduring memories in adulthood. For example, recent studies have shown that prenatal- hippocampal LTP. Stimulation of $\alpha-7$ nAChRs can rescue memory deficits in animal models of Alzheimer's disease (Chen et al. 2006) as well as improve working and recognition memory in normal subjects (Boess et al. 2007).

In conclusion, the present results demonstrate the sensitivity of timing, emotion, and memory performance (TEMP) to prenatal choline availability. The reduction of DRL-related burst responding induced by prenatal-choline supplementation suggests that the amount of dietary choline supplied during gestation is able to regulate behavioral inhibition in adult offspring. Future studies should determine the extent to which these beneficial effects generalize to other aspects of emotional behavior. The findings that SUP-male rats were less efficient than CON-male rats and that SUP-female rats were more efficient than CONfemale rats in acquiring longer DRL criterion times suggests that there is an interaction between prenatal-choline supplementation and gonadal steroids. While the underlying organizational and/or activational mechanisms for this interaction remain to be determined (Williams et al. 1990; Williams and Meck 1991), it is known that some aspects of choline metabolism are sexually dimorphic and estrogen sensitive (Fisher et al. 2007). Finally, prenatal-choline supplementation appears to enable subjects to form temporal memories that are more precise and enduring based on the observation that both SUP-male and SUP-female rats displayed a high degree of temporal control of their IRT distributions, albeit with SUP-males displaying a lower response efficiency compared with CON-male rats as a result of the persistence of their previously trained response repertoires.

\section{Materials and Methods}

\section{Animals and diet}

Forty timed-pregnant Sprague-Dawley rats (CD strain, Charles River Laboratories) were used to generate four groups of adult offspring randomly selected from at least 16 litters that were composed of CON-female, SUP-female, CON-male, and SUPmale treatment groups ( $n=8$ /group). Upon arrival in the colony on day nine of gestation (ED 9), all dams had ad lib access to water and a choline sufficient purified diet (AIN76A with $1.1 \mathrm{~g} / \mathrm{kg}$ choline chloride substituted for choline bitartrate; Dyets, Inc.) throughout pregnancy except ED 12-17. During ED 12-17, half of the dams $(n=8)$ received a version of the purified diet that contained choline chloride $\sim 4.5$ times higher (e.g., $5.0 \mathrm{~g} / \mathrm{kg}$ ) than the amount of choline chloride in the sufficient diet. The other half of the dams $(n=8)$ continued to receive the sufficient diet throughout the period of ED 12-17. Thus, choline supplementation was only conducted during the time frame of ED 12-17, 
because previous evidence has been shown that treatment during this developmental period can cause a lasting improvement in memory function in adulthood (e.g., Meck and Williams 2003).

At birth, all pups were randomly divided among untreated foster mothers with litters evenly divided by sex and choline treatments. All pups were weaned at postnatal day 24 , after which the randomly chosen 32 rats were housed in same-sex pairs and given free access to water and the purified choline sufficient diet as previously described. At about 7 mo of age and $1 \mathrm{wk}$ before behavioral training, all of the rats were food restricted until they reached $85 \%$ of free-feeding body weight. From then on, a daily ration of a control diet was given to rats after each behavioral session. Throughout the experiment, the colony room was maintained at $21^{\circ} \mathrm{C}$ and at a 12 -h light-dark cycle with the lights on at 07:00 h. The housing conditions and the experiments were in accordance with protocols approved by the Institutional Animal Care and Use Committee of Duke University.

\section{Apparatus}

All experimental data were obtained in eight identical lever boxes (MED Associates, Inc., Model ENV-007) housed in light and sound-attenuating chambers equipped with eyepiece viewers for observation and ventilation fans (providing the $60-\mathrm{dB}$ sound). Each lever box had inside dimensions of $24 \mathrm{~cm} \times 31 \mathrm{~cm} \times 31$ $\mathrm{cm}$. The top, side walls, and door were constructed of clear acrylic plastic. The front and back walls were constructed of stainless steel, and the floor was comprised of 19 parallel stainless-steel bars. Each lever box was equipped with two response levers (MED Associates, Inc., model ENV-112) situated on the front wall of the lever box. Precision food pellets (45 mg; Research Diets, Inc.) could be delivered by a pellet dispenser (MED Associates, Inc., model ENV-203) to a food cup on the front wall, $1 \mathrm{~cm}$ above the floor. A photobeam sensor (MED Associates, Inc., model ENV254) that serves as a head entry detector was connected to the wall that encloses the food cup tray. A $28-\mathrm{V}, 80-\mathrm{mA}, 5000-1 \mathrm{x}$ house light was mounted at the center, top, of the front wall and could be used to illuminate the box. A white noise amplifier/ speaker system (MED Associates, Inc., model ENV-225) was mounted on the opposite wall from the levers, but was not used in the current study. An IBM-PC compatible computer attached to an electronic interface (MED Associates, Inc., models DIG-700 and SG-215) was used to control the experimental equipment and to record the data.

\section{Experimental procedures}

\section{Pretraining}

All rats received five sessions of combined magazine and lever training (autoshaping) followed by eight sessions of variableratio 2 (VR-2) training. During autoshaping sessions, a food pellet was delivered every minute for $60 \mathrm{~min}$, and one of the two side levers was randomly assigned to each rat as an effective lever throughout the study. The other side lever served as a control lever that had no programmed result. Pressing the effective lever once delivered a food pellet. The autoshaping session ended for each rat when 60 food pellets were obtained by either of the two methods described above. During VR-2 training, which lasted 30 min, only lever presses delivered food pellets, and the reinforcement contingency was set to a mean of two lever presses with a range of from 1 to 4 . All pretraining/training sessions were conducted $7 \mathrm{~d} /$ wk from 7 p.m. to 11 p.m.

\section{DRL training}

In the DRL schedule, the reinforcement contingency was specified such that lever presses separated by an inter-response time (IRT) equal or greater than the criterion time would be reinforced. For example, in the DRL 5-sec schedule training, pressing the lever at least $5 \mathrm{sec}$ after the previous lever press (IRT $\geq 5 \mathrm{sec}$ ) yielded the delivery of a food pellet and the reset of the experimental clock. Pressing the lever less than $5 \mathrm{sec}$ after the previous lever press simply reset the experimental clock and required the rat to wait at least another $5 \mathrm{sec}$ before having the opportunity to earn a food pellet. This general principle applied to all of the DRL training stages from 5 to $72 \mathrm{sec}$. The session length was $30 \mathrm{~min}$ for the DRL 5-sec, DRL 10-sec, and DRL 36-sec schedules and the session length was 60 min for the DRL 36-sec and DRL 72-sec schedules. There were eight training sessions for the DRL 5-sec schedule, 16 sessions for the DRL 10-sec schedule, and at least 60 sessions for the DRL 18-sec, DRL 36-sec, and DRL 72-sec schedules. Beginning with the DRL 18-sec schedule, we applied a training threshold, such that once all rats in a group achieved a specified level of modified response efficiency $(>0.40)$, this group would receive 10 additional training sessions and then be shifted to the next DRL schedule. Only the SUP-female rats achieved this threshold during DRL 36-sec schedule training, and consequently were shifted to the DRL 72-sec schedule following 28 sessions of DRL 36-sec schedule training.

\section{Nutrients}

Two purified diets were used. The choline sufficient diet (CONAIN-76A with choline chloride substituted for choline bitartrate; Dyets, Inc.) contained $1.1 \mathrm{~g} / \mathrm{kg}$ choline chloride, while the choline supplemented diet (SUP) was the same purified diet with 5 $\mathrm{g} / \mathrm{kg}$ choline chloride. During ED 12-17, half of the pregnant dams had their diet switched to the SUP diet and half remained on the CON diet (see Animals and Diet section).

\section{Data / statistical analyses}

During each DRL training stage, modified response efficiency (ratio between reinforcement rate and overall response rate after the subtraction of burst responses) and burst responses (lever presses that occur within $2 \mathrm{sec}$ of a previous lever press; i.e., IRT $<2 \mathrm{sec}$ ) were indexed as a function of training session. In order to highlight the process of acquiring DRL 18-sec, DRL 36-sec, and DRL 72 -sec schedules, the performance of three sessions during early (e.g., sessions 1-3), intermediate (e.g., sessions 29-31), and late (e.g., sessions 58-60) phases of training was pooled into three different levels of the training factor for subsequent analysis of variance (ANOVA). Consequently, 3-way ANOVAs were conducted on sex, prenatal choline treatment, and training phase for the index of modified response efficiency and burst responding. Post-hoc comparisons were conducted by using Tukey's honestly significant difference (HSD) tests. The $P$-value was set at 0.05 for all statistical analyses. Finally, we used a sliding time-window method to determine the peak time of the IRT distribution for each rat as a function of sessions as previously described (e.g., Cheng et al. 2006a). This method of identifying the peak time allowed us to infer when rats maximally expected reinforcement to occur.

\section{Acknowledgments}

We thank Melissa Glenn for her advice and thoughtful comments on an earlier version of this manuscript. This research was financially supported by the National Institute of Aging grant AG009525.

\section{References}

Alkondon, M., Pereira, E.F., Cortes, W.S., Maelicke, A., and Albuquerque, E.X. 1997. Choline is a selective agonist of $\alpha-7$ nicotinic acetylcholine receptors in rat brain neurons. Eur. J. Neurosci. 9: 2734-2742.

Archer, J. 1977. Testosterone and persistence in mice. Anim. Behav. 25: $479-488$.

Bardo, M.T., Cain, M.E., and Bylica, K.E. 2006. Effect of amphetamine on response inhibition in rats showing high or low response to novelty. Pharmacol. Biochem. Behav. 85: 98-104.

Beatty, W.W. 1973. Effects of gonadectomy on sex differences in DRL behaviour. Physiol. Behav. 10: 177-178.

Boess, F.G., De Vry, J., Erb, C., Flessner, T., Hendrix, M., Luithle, J., Methfessel, C., Riedl, B., Schnizler, K., van der Staay, F.J., et al. 2007. The novel $\alpha 7$ nicotinic acetylcholine receptor agonist N-[(3R)-1-azabicyclo[2.2.2]oct-3-yl]-7-[2-(methoxy)phenyl]1-benzofuran-2-carboxamide improves working and recognition memory in rodents. J. Pharmacol. Exp. Ther. 321: 716-725. 
Buhusi, C.V. and Meck, W.H. 2005. What makes us tick? Functional and neural mechanisms of interval timing. Nat. Rev. Neurosci. 6: $755-765$.

Cermak, J.M., Blusztajn, J.K., Meck, W.H., Williams, C.L., Fitzgerald, C.M., Rosene, D.L., and Loy, R. 1999. Prenatal availability of choline alters the development of acetylcholinesterase in rat hippocampus. Dev. Neurosci. 21: 94-104.

Chen, L., Yamada, K., Nabeshima, T., and Sokabe, M. 2006. $\alpha 7$ nicotinic acetylcholine receptor as a target to rescue deficit in hippocampal LTP induction in $\beta$-amyloid infused rats. Neuropharmacology 50: $254-268$

Cheng, R.K. and Meck, W.H. 2007. Prenatal choline supplementation increases sensitivity to time by reducing non-scalar sources of variance in adult temporal processing. Brain Res. 1186: 242-254.

Cheng, R.K., MacDonald, C.J., and Meck, W.H. 2006a. Differential effects of cocaine and ketamine on time estimation: Implications for neurobiological models of interval timing. Pharmacol. Biochem. Behav. 85: 226-233.

Cheng, R.K., Meck, W.H., and Williams, C.L. 2006b. $\alpha 7$ nicotinic acetylcholine receptors and temporal memory: Synergistic effects of combining prenatal choline and nicotine on reinforcement-induced resetting of an interval clock. Learn. Mem. 13: 127-134.

Cheng, R.K., Ali, Y.M., and Meck, W.H. 2007a. Ketamine "unlocks" the reduced clock-speed effect of cocaine following extended training: Evidence for dopamine-glutamate interactions in timing and time perception. Neurobiol. Learn. Mem. 88: 149-159.

Cheng, R.K., Hakak, O.L., and Meck, W.H. 2007b. Habit formation and the loss of control of an internal clock: Inverse relationship between the level of baseline training and the clock-speed enhancing effects of methamphetamine. Psychopharmacology 193: 351-362.

Church, R.M., Lacourse, D.M., and Crystal, J.D. 1998. Temporal search as a function of the variability of interfood intervals. J. Exp. Psychol. Anim. Behav. Process. 24: 291-315.

Cohen, C., Perrault, G., and Sanger, D.J. 1997. Assessment of the antidepressant-like effects of L-type voltage-dependent channel modulators. Behav. Pharmacol. 8: 629-638.

Dallal, N.L. and Meck, W.H. 1990. Hierarchical structures: Chunking by food type facilitates spatial memory. J. Exp. Psychol. Anim. Behav. Process. 16: 69-84.

Droit-Volet, S. and Meck, W.H. 2007. How emotions colour our perception of time. Trends Cogn. Sci. 11: 504-513.

Evenden, J.L. 1999. Varieties of impulsivity. Psychopharmacology 146: $348-361$.

Fisher, L.M., daCosta, K.A., Kwock, L., Stewart, P.W., Lu, T.S., Stabler, S.P., Allen, R.H., and Zeisel, S.H. 2007. Sex and menopausal status influence human dietary requirements for the nutrient choline. Am. J. Clin. Nutr. 85: 1275-1285.

Glenn, M.J., Gibson, E.M., Kirby, E.D., Mellott, T.J., Blusztajn, J.K., and Williams, C.L. 2007. Prenatal choline availability modulates hippocampal neurogenesis and neurogenic responses to enriching experiences in adult female rats. Eur. J. Neurosci. 25: 2473-2482.

Holmes, G.L., Yang, Y., Liu, Z., Cermak, J.M., Sarkisian, M.R., Stafstrom, C.E., Neill, J.C., and Blusztajn, J.K. 2002. Seizure-induced memory impairment is reduced by choline supplementation before or after status epilepticus. Epilepsy Res. 48: 3-13.

Jones, J.P., Meck, W.H., Williams, C.L., Wilson, W.A., and Swartzwelder, H.S. 1999. Choline availability to the developing rat fetus alters adult hippocampal long-term potentiation. Dev. Brain Res. 118: $159-167$.

Louis, C., Cohen, C., Depoortere, R., and Groebel, G. 2006. Antidepressant-like effects on the corticotropin-releasing factor I receptor antagonist, SSRI25543, and the vasopressinb Ib receptor antagonist, SSRI49415, in a DRL-72s schedule in the rat. Neuropsychopharmacology 31: 2180-2187.

MacDonald, C.J. and Meck, W.H. 2004. Systems-level integration of interval timing and reaction time. Neurosci. Biobehav. Rev. 28: $747-769$.

Matell, M.S. and Meck, W.H. 2000. Neuropsychological mechanisms of interval timing behaviour. Bioessays 22: 94-103.

Matell, M.S. and Meck, W.H. 2004. Cortico-striatal circuits and interval timing: Coincidence-detection of oscillatory processes. Cogn. Brain Res. 21: $139-170$.

Matell, M.S. and Portugal, G.S. 2007. Impulsive responding on the peak-interval procedure. Behav. Processes 74: 198-208.

McAuley, J.D., Miller, J.P., and Pang, K.C. 2006. Modelling the effects of the NMDA receptor antagonist MK-801 on timing in rats. Behav. Neurosci. 120: 1163-1168.

Meck, W.H. 1983. Selective adjustment of the speed of internal clock and memory processes. J. Exp. Psychol. Anim. Behav. Process. 9: 171-201.

Meck, W.H. 1988. Hippocampal function is required for feedback control of an internal clock's criterion. Behav. Neurosci. 102: 54-60.
Meck, W.H. 1996. Neuropharmacology of timing and time perception. Cogn. Brain Res. 3: 227-242.

Meck, W.H. 2002. Choline uptake in the frontal cortex is proportional to the absolute error of a temporal memory translation constant in mature and aged rats. Learn. Motiv. 33: 88-104.

Meck, W.H. and MacDonald, C.J. 2007. Amygdala inactivation reverses fear's ability to impair divided attention and make time still. Behav. Neurosci. 121: 707-720.

Meck, W.H. and Williams, C.L. 1997a. Characterization of the facilitative effects of perinatal choline supplementation on timing and temporal memory. NeuroReport 8: 2831-2835.

Meck, W.H. and Williams, C.L. 1997b. Perinatal choline supplementation increases the threshold for chunking in spatial memory. NeuroReport 8: 3053-3059.

Meck, W.H. and Williams, C.L. 1997c. Simultaneous temporal processing is sensitive to prenatal choline availability in mature and aged rats. NeuroReport 8: 3045-3051.

Meck, W.H. and Williams, C.L. 1999. Choline supplementation during prenatal development reduces proactive interference in spatial memory. Dev. Brain Res. 118: 51-59.

Meck, W.H. and Williams, C.L. 2003. Metabolic imprinting of choline by its availability during gestation: Implications for memory and attentional processing across the lifespan. Neurosci. Biobehav. Rev. 27: 385-399.

Meck, W.H. and Williams, C.L. 2008. Organizational effects of perinatal choline supplementation on spatial exploration as a function of sex, time of day, and aging. Neurobiol. Aging (in press).

Meck, W.H., Church, R.M., and Wenk, G.L. 1986. Arginine vasopressin inoculates against age-related increases in sodium-dependent high affinity choline uptake and discrepancies in the content of temporal memory. Eur. J. Pharmacol. 130: 327-331.

Meck, W.H., Church, R.M., Wenk, G.L., and Olton, D.S. 1987. Nucleus basalis magnocellularis and medial septal area lesions differentially impair temporal memory. J. Neurosci. 7: 3505-3511.

Meck, W.H., Smith, R.A., and Williams, C.L. 1988. Pre- and postnatal choline supplementation produces long-term facilitation of spatial memory. Dev. Psychobiol. 21: 339-353.

Meck, W.H., Smith, R.A., and Williams, C.L. 1989. Organizational changes in cholinergic activity and enhanced visuospatial memory as a function of choline administered prenatally or postnatally or both. Behav. Neurosci. 103: 1234-1241.

Mellott, T.J., Williams, C.L., Meck, W.H., and Blusztajn, J.K. 2004. Prenatal choline supplementation advances hippocampal development and enhances MAPK and CREB activation. FASEB $J$. 18: $545-547$.

Molina-Hernandez, M., Contreras, C.M., and Tellez-Alcantara, P. 2000. Antidepressant-like effects of pregnancy and progesterone in Wistar rats as measured in the differential reinforcement of the low-rate 72 s task. Psychopharmacology 151: 306-311.

Montoya, D.A.C., White, A.M., Williams, C.L., Blusztajn, J.K., Meck, W.H., and Swartzwelder, H.S. 2000. Prenatal choline exposure alters hippocampal responsiveness to cholinergic stimulation in adulthood. Dev. Brain Res. 123: 25-32.

Nevin, J.A., McLean, A.P., and Grace, R.C. 2001. Resistance to extinction: Contingency termination and generalization decrement. Anim. Learn. Behav. 29: 176-191.

Paule, M.G., Meck, W.H., McMillan, D.E., Bateson, M., Popke, E.J., Chelonis, J.J., and Hinton, S.C. 1999. The use of timing behaviors in animals and humans to detect drug and/or toxicant effects. Neurotoxicol. Teratol. 21: 491-502.

Pyapali, G.K., Turner, D.A., Williams, C.L., Meck, W.H., and Swartzwelder, H.S. 1998. Prenatal dietary choline supplementation decreases the threshold for induction of long-term potentiation in young adult rats. J. Neurophysiol. 79: 1790-1796.

Richards, J.B. and Seiden, L.S. 1991. A quantitative interresponse-time analysis of DRL performance differentiates similar effects of the antidepressant desipramine and the novel anxiolytic gepirone. $J$. Exp. Anal. Behav. 56: 173-192.

Richards, J.B., Sabol, K.E., and Seiden, L.S. 1993. DRL interresponse-time distributions: Quantification by peak deviation analysis. J. Exp. Anal. Behav. 60: 361-385.

Richards, J.B., Sabol, K.E., Hand, T.H., Jolly, D.C., Marek, G.J., and Seiden, L.S. 1994. Buspirone, gepirone, ipsapirone, and zalospirone have distinct effects on the differential-reinforcement-of-low-rate 72-s schedule when compared with 5-HTP and diazepam. Psychopharmacology 114: 39-46.

Ripley, T.L., Horwood, J.M., and Stephens, D.N. 2001. Evidence for impairment of behavioural inhibition in performance of operant tasks in tPA - / - mice. Behav. Brain Res. 125: 215-227.

Ross, L. and Santi, A. 2000. The effects of estrogen on temporal and numerical processing in ovariectomized female rats. Psychobiology 28: $394-405$. 
Schulz, D., Topic, B., De Souza Silva, M.A., and Huston, J.P. 2004. Extinction-induced immobility in the water maze and its neurochemical concomitants in aged and adult rats: A possible model for depression? Neurobiol. Learn. Mem. 82: 128-141.

Tropp, J. and Markus, E.J. 2001. Sex differences in the dynamics of cue utilization and exploratory behavior. Behav. Brain Res. 119: 143-154.

van den Bergh, F.S., Bloemarts, E., Chan, J.S., Groenink, L., Olivier, B., and Oosting, R.S. 2006. Spontaneously hypertensive rats do not predict symptoms of attention-deficit hyperactivity disorder. Pharmacol. Biochem. Behav. 83: 380-390.

van Hest, A., van Haaren, F., and van de Poll, N.E. 1987. Behavioral differences between male and female wistar rats on DRL schedules: Effects of stimuli promoting collateral activities. Physiol. Behav. 39: $255-261$.

Wiley, J.L., Compton, A.D., and Golden, K.M. 2000. Separation of drug effects on timing and behavioral inhibition by increased stimulus control. Exp. Clin. Psychopharmacol. 8: 451-461.

Williams, C.L. and Meck, W.H. 1991. Organizational effects of gonadal steroids on sexually dimorphic spatial ability.
Psychoneuroendocrinology 16: 157-177.

Williams, C.L., Barnett, A.M., and Meck, W.H. 1990. Organizational effects of early gonadal secretions on sexual differentiation in spatial memory. Behav. Neurosci. 104: 84-97.

Williams, C.L., Meck, W.H., Heyer, D., and Loy, R. 1998. Hypertrophy of basal forebrain neurons and enhanced visouspatial memory in perinatally choline-supplemented rats. Brain Res. 794: 225-238.

Yang, Y., Liu, Z., Cermak, J.M., Tandon, P., Sarkisian, M.R., Stafstrom, C.E., Neill, J.C., Blusztajn, J.K., and Holmes, G.L. 2000. Protective effects of prenatal choline supplementation on seizure-induced memory impairment. J. Neurosci. 20: RC109. http://www.jneurosci. org/cgi/content/full/20/22/RC109?ck=nck.

Zeisel, S.H. and Niculescu, M.D. 2006. Perinatal choline influences brain structure and function. Nutr. Rev. 64: 197-203.

Received August 6, 2007; accepted in revised form December 29, 2007. 

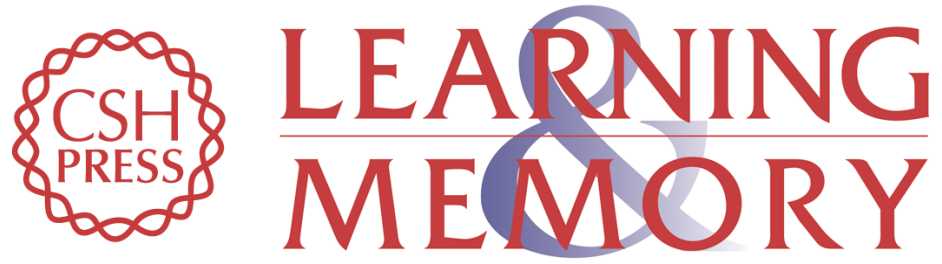

Prenatal choline supplementation alters the timing, emotion, and memory performance (TEMP) of adult male and female rats as indexed by differential reinforcement of low-rate schedule behavior

Ruey-Kuang Cheng, Christopher J. MacDonald, Christina L. Williams, et al.

Learn. Mem. 2008, 15:

Access the most recent version at doi:10.1101//m.729408

References This article cites 63 articles, 5 of which can be accessed free at: http://learnmem.cshlp.org/content/15/3/153.full.html\#ref-list-1

License

Email Alerting

Receive free email alerts when new articles cite this article - sign up in the box at the Service top right corner of the article or click here. 